\title{
Redes Neurais Artificiais para a estimação da densidade básica da madeira
}

\section{Artificial neural networks for basic wood density estimation}

\author{
Hélio Garcia Leite ${ }^{1}$, Daniel Henrique Breda Binoti², \\ Ricardo Rodrigues de Oliveira Neto ${ }^{3}$, Pablo Falco Lopes ${ }^{4}$, \\ Rodrigo Ribeiro de Castro ${ }^{5}$, Erik Júnior Paulino ${ }^{6}$, \\ Mayra Luiza Marques da Silva Binoti ${ }^{7}$ Jorge Luiz Colodette ${ }^{8}$
}

\begin{abstract}
Resumo
A densidade da madeira é a propriedade que mais se correlaciona com as outras propriedades físicas e mecânicas, sendo usada para definir o uso apropriado da madeira industrialmente. Diversas ferramentas computacionais e matemáticas são utilizadas para modelar a densidade básica dos povoamentos, destacando-se as Redes Neurais Artificiais (RNA). Os dados foram provenientes de 2247 árvores de 20 clones de Eucalyptus ssp.. em 6 arranjos espaciais, com idades variando entre 3 e 6 anos. As RNA foram treinadas visando a estimação da densidade na idade de corte (6 anos), com variáveis de entrada como idade (anos), área basal $\left(\mathrm{m}^{2} / \mathrm{ha}\right)$, incremento médio anual $\left(\mathrm{m}^{3} / \mathrm{ha} / \mathrm{ano}\right)$ na idade de medição, altura total $(\mathrm{m})$, diâmetro a 1,3 $\mathrm{m}$ da superfície do solo (Dap) $(\mathrm{cm})$, número de fustes por hectare $(\mathrm{n} / \mathrm{ha})$ e a razão entre dap, altura total e interação entre as variáveis. Os algoritmos de treinamento foram error backpropagation, resilient propagation, Manhattan update rule, scaled conjugate gradient, levenberg marquardt, quick propagation, simulated annealing, e algoritmos genéticos. As estimativas foram avaliadas com base nos coeficientes de correlação com os valores observados, raiz quadrada do erro médio percentual (RMSE), análise gráfica dos resíduos (erros percentuais) e histograma de frequência percentual dos erros percentuais. As melhores configurações foram a de 4 neurônios de camada oculta e função de ativação tangente hiperbólica na camada oculta e sigmoide na camada de saída e a com 8 neurônios na camada oculta, com função de ativação tangente hiperbólica na camada oculta e sigmoide na camada de saída.
\end{abstract}

Palavras-chave: Parametrização, algoritmos, planejamento operacional.

\footnotetext{
Abstract

The Wood density is the property which correlates most closely with the other physical and mechanical properties and is used to set the appropriate use of wood industrially. Several computational and mathematical tools are used to model the basic density of stands, highlighting the Artificial Neural Networks (ANN). Data came from 2247 trees of 20 clones of Eucalyptus spp. in 6 spatial arrangements, aged between 3 and 6 years. The RNA's were trained in order to estimate the density at the cutting age (6 years), with input variables such as age (years), basal area $\left(\mathrm{m}^{2} / \mathrm{ha}\right)$, mean annual increment $\left(\mathrm{m}^{3} /\right.$ ha / year) of measuring age , total height $(\mathrm{m})$, diameter at $1.3 \mathrm{~m}$ from the soil surface (Dap) $(\mathrm{cm})$, stems number per hectare $(\mathrm{n} / \mathrm{ha})$ and the ratio of dbh, total height and interaction between variables. Training algorithms were: error backpropagation, resilient propagation, Manhattan update rule, scaled conjugate gradient, Levenberg Marquardt, quick propagation, simulated annealing, and genetic algorithms. The estimates were evaluated

1Professor Titular do Departamento de Engenharia Florestal. UFV - Universidade Federal de Viçosa / Centro de Ciências Agrárias. Caixa-postal: 36570000 - 36570-000 - Vicosa, MG, Brasil. E-mail: hgleite@ufv.br

${ }^{2}$ Doutor em Ciência Florestal. UFV - Universidade Federal de Viçosa / Centro de Ciências Agrárias. Caixa-postal: 36570000 - 36570-000 - Vicosa, MG, Brasil. E-mail: danielhbbinoti@gmail.com

${ }^{3}$ Mestrando Ciência Florestal. UFV - Universidade Federal de Viçosa / Centro de Ciências Agrárias. Caixa-postal: 36570000 - 36570-000 - Vicosa, MG, Brasil. E-mail: engricardoneto@me.com

${ }^{4}$ Mestre Ciência Florestal. UFV - Universidade Federal de Viçosa / Centro de Ciências Agrárias. Caixa-postal: 36570000 36570-000 - Vicosa, MG, Brasil. E-mail: pfalcol@dapflorestal.com.br

${ }^{5}$ Doutorando em Ciência Florestal. UFV - Universidade Federal de Viçosa / Centro de Ciências Agrárias. Caixa-postal: 36570000 - 36570-000 - Vicosa, MG, Brasil.

${ }^{6}$ Mestre em Ciência Florestal. UFVJM - Universidade Federal dos Vales do Jequitinhonha e Mucuri. Caixa Postal 58 - 39100 000 - Diamantina, MG, Brasil.

${ }^{7}$ Professora Adjunta II do Departamento de Ciências Florestais e da Madeira. UFES - Universidade Federal do Espírito Santo / Centro de Ciências Agrárias. Av. Governador Lindemberg, 316 Centro - 29550000 - Jerônimo Monteiro, ES, Brasil. E-mail: mayrabinoti@gmail.com

${ }^{8}$ Professor Titular. UFV - Universidade Federal de Viçosa / Centro de Ciências Agrárias. Caixa-postal: 36570000 - $36570-000$ - Vicosa, MG, Brasil. E-mail: danielhbbinoti@gmail.com
} 
according to the correlation coefficients with the observed values, square root of the average percentage error (RMSE), graphic analysis of residues (percentage error) and histogram percentage frequency of the percentage errors. The best settings were 4 neurons in the hidden layer and hyperbolic tangent activation function in the hidden sigmoid layer and the output layer and 8 neurons in the hidden layer, hyperbolic tangent activation function in the hidden layer and the output layer sigmoid.

Keywords: Parameterization, algorithms, operational planning.

\section{INTRODUÇÃO}

A densidade da madeira é uma das propriedades mais importantes, na maioria dos usos da madeira, uma vez que se correlaciona bem com muitas outras propriedades físicas e mecânicas (TSOUMIS, 1991; KNAPIC et al., 2007). A densidade da madeira é dependente do tamanho das células e da espessura das paredes das células, e da inter-relação entre o número de células de vários tipos, em termos de tamanho e espessura das paredes (PANSHIN; ZEEUW, 1980).

A densidade da madeira apresenta uma grande variabilidade natural, sendo influenciada pelo ritmo de crescimento das árvores, pela qualidade do local e condições ambientais (MITCHELL, 1958). Devido a sua grande importância, a densidade influencia na definição do uso industrial apropriado da madeira (VITAL; VALENTE, 1986; SANTOS; SANSÍGOLO, 2007; MOKFIENSKI et al., 2008, DIAS; LAHR, 2004; BELINI et al., 2007). O inventário Florestal pode ser utilizado como ferramenta para a quantificação e estimação da densidade.

O inventário florestal é realizado por inferências qualitativas e quantitativas sobre os povoamentos florestais. Tradicionalmente, o volume dos inventários florestais é expresso em metros cúbicos, porém, quando o povoamento destina-se a produção de celulose, painéis ou energia, a produção florestal deveria ser expressa em massa de madeira, que é estimada em função da densidade básica da madeira (CAMPOS; LEITE, 2013). Contudo, devido à variabilidade natural apresentada pela densidade básica, sua estimação, em função de parâmetros do povoamento, é uma tarefa complexa.

Diversas ferramentas computacionais e de modelagem matemática tem sido utilizadas com êxito em diversas situações no setor florestal. Dentre essas ferramentas destacam-se as Redes Neurais Artificiais (RNA). As RNA são um sistema de computação constituído por um número de elementos de processamento simples, altamente interligadas, para a realização de determinada tarefa. Diversos trabalhos têm sido desenvolvidos visando a adaptação e parametrização de técnicas de RNA, para diversas situações como, a estimação do volume de árvores (SILVA et al., 2008), crescimento e produção (BINOTI, 2010) e taper (LEITE et al., 2010), dentre outras aplicações.

O objetivo deste estudo foi modelar a densidade básica de povoamentos de clones de eucalipto em função de variáveis numéricas, obtidas pelo inventário florestal contínuo (IFC) empregando redes neurais artificiais.

\section{MATERIAL E MÉTODOS}

\section{Dados}

Os dados utilizados neste estudo foram provenientes de 2247 árvores amostras. Os plantios estão localizados nos estados de São Paulo e Minas Gerais. As árvores amostras foram obtidas de 20 clones de Eucalyptus spp., distribuídos em seis diferentes arranjos espaciais $(2,50 \times 1,80 ; 2,80 \times 3,20$; $3,00 \times 1,50 ; 3,00 \times 2,00 ; 3,00 \times 3,00 ; 4,00 \times 1,10)$ abrangendo todo a variação de clones e espaçamento. Uma descrição da variação da densidade observada, e das variáveis obtidas no inventário, são apresentadas na Tabela 1 . O número de medições variou de uma a cinco idades, compreendendo 170 parcelas.

Os dados, englobando todas as medições disponíveis, foram divididos aleatoriamente em dois conjuntos, um para o treinamento das redes (134 parcelas) e outro para validar as redes treinadas (34 parcelas), com uma proporção de cerca de $80 \%$ dos dados para treinamento e $20 \%$ para generalização.

Os valores que extrapolaram a tendência geral, das variáveis utilizadas em função da idade, não foram eliminados, a fim de verificar a capacidade das redes neurais artificiais em lidar com outliers ou ruídos. 
Tabela 1. Valores mínimo, médios, máximos e o desvio padrão do Incremento Médio Anual ( $\left.\mathrm{m}^{3} / \mathrm{ha} / \mathrm{ano}\right)$, Idade (anos), Área Basal $\left(\mathrm{m}^{2} / \mathrm{ha}\right)$, Altura Total $(\mathrm{m})$, Dap $(\mathrm{cm})$ e Densidade Básica $\left(\mathrm{g} / \mathrm{cm}^{3}\right)$ dos dados do estudo.

Table 1. Minimum, mean, maximum and standard deviation of the Mean Annual Increment ( $\left.\mathrm{m}^{3} / \mathrm{ha} / \mathrm{year}\right)$, age (years), Basal Area $\left(\mathrm{m}^{2} / \mathrm{ha}\right)$ Total.

\begin{tabular}{lcccc}
\hline Parâmetro & Mínimo & Média & Máximo & Desvio Padrão \pm \\
\hline Incremento Médio Anual (m³/ha/ano) & 19,81 & 48,59 & 72,55 & 10,21 \\
Idade (anos) & 2,04 & 5,03 & 7,83 & 1,60 \\
Área Basal $\left(\mathrm{m}^{2} / \mathrm{ha}\right)$ & 8,7000 & 22,9741 & 33,4667 & 4,7517 \\
Altura Total $(\mathrm{m})$ & 11,17 & 23,26 & 30,45 & 4,70 \\
Dap $(\mathrm{cm})$ & 8,33 & 14,83 & 18,40 & 2,25 \\
Número de Fustes $(\mathrm{n} / \mathrm{ha})$ & 870 & 1368 & 2415 & 310 \\
Densidade Básica $\left(\mathrm{g} / \mathrm{cm}^{3}\right)$ & 0,3467 & 0,4349 & 0,5186 & 0,0423 \\
\hline
\end{tabular}

\section{Redes Neurais Artificiais (RNA)}

As RNA foram treinadas visando a estimação da densidade na idade de corte ( 6 anos), a partir de informações facilmente obtidas em qualquer idade do povoamento. As variáveis utilizadas, como input, foram: idade (anos), área basal ( $\left.\mathrm{m}^{2} / \mathrm{ha}\right)$, incremento médio anual ( $\left.\mathrm{m}^{3} / \mathrm{ha} / \mathrm{ano}\right)$ na idade de medição, altura total $(\mathrm{m})$, diâmetro a 1,3 $\mathrm{m}$ da superfície do solo (Dap) (cm), número de fustes por hectare (n/ha) e a razão entre dap e altura total. Considerou-se apenas variáveis contínuas para a estimação da densidade básica na idade de corte.

As variáveis numéricas foram normalizadas linearmente no intervalo de 0 a 1 (GOLDSCHMIDT; PASSOS, 2005; HEATON, 2011).

A camada de entrada foi constituída de 7 neurônios, sendo um para cada variável numérica e 1 neurônio com entrada fixa de valor 1, referente ao limiar de excitação. Como saída, utilizou-se a densidade básica obtida na idade de corte. As redes foram constituídas de apenas uma camada oculta, sendo que o número de neurônios nessa camada variou de 1 a 8 (que corresponde ao número de neurônios na camada de entrada). Como funções de ativação da camada oculta e de saída utilizou-se as funções: linear, log, tangente hiperbólica, seno e sigmoide (HEATON, 2011).

Os algoritmos de treinamentos utilizados foram error backpropagation, resilient propagation, Manhattan update rule, scaled conjugate gradient, levenberg marquardt, quick propagation, simulated annealing, e algoritmos genéticos (HEATON, 2011).

Para o algoritmo backpropagation utilizou-se variações no valor da taxa de aprendizado de 0,001 a 1,000 (a cada 0,001), bem como variações no parâmetro momentum de 0,0000 a 1,000 (a cada 0,0005), totalizando 1000 valores de taxas de aprendizado e 2001 valores de momentum. Os valores de taxa de aprendizado e taxas de momentum foram combinados totalizando 2.001 .000 combinações. Além dessas combinações utilizou-se a estratégia de auto ajuste dos valores de taxa de aprendizado e momentum, processo que reduz os valores destes parâmetros com a evolução do algoritmo, resultando em 8.004.000 configurações do algoritmo backpropagation.

Para o algoritmo resilient propagation, considerou-se quatro variações básicas denominadas RPROP+, RPROP-, iRPROP+, iPROP (HEATON, 2011). No algoritmo Manhattan update rule, que por definição necessita de pequenas taxas de treinamento, restringiu-se a utilização de somente um valor de taxa de aprendizado: 0,00001. Os algoritmos scaled conjugate gradient, levenberg marquardt apresentam somente uma configuração não dependendo de valores de parâmetros de treinamento.

O algoritmo de treinamento conhecido como quick propagation baseia-se no método de Newton que é utilizado para obtenção de raízes de uma função. Esse algoritmo é tolerante a altas taxas de aprendizado, por isso avaliou-se taxas variando de 0,1 a 10,0 (a cada 0,1) totalizando 100 configurações desse algoritmo.

A metaheurística Simulated annealing baseia-se no processo de recozimento de metais utilizado em processos metalúrgicos, o processo consiste em aquecer um metal e verificar como esse metal se resfria e congela numa estrutura cristalina de energia mínima. Como principais parâmetros desse algoritmo tem-se o número de ciclos por interação e os valores de temperatura máxima e mínima. Os valores de números de ciclos avaliados foram variados de 100 a 1000 (a cada 100), com temperatura mínima de dois e máxima de dez, totalizando 10 configurações.

Outra metaheurística utilizada foi o Algoritmo Genético (AG). O AG baseia-se no processo de evolução natural das espécies e nos princípios de reprodução genética. O sistema possui, como principais parâmetros configuráveis, o tamanho da população, a probabilidade de mutação e a taxa 
de cruzamento. Testou-se valores de população variando de 50 a 1000 (a cada 50), totalizando 20 valores de população, valores de mutação variando de 0,1 a 0,5 (a cada 0,1 ), totalizando 5 valores de mutação, valores de cruzamento, variando 0,05 a 0,70 (a cada 0,05) totalizando 14 valores. Foram testados, no total, 114 configurações de AG.

Foram avaliadas todas as possíveis combinações entre números de neurônios na camada oculta, tipo de funções de ativação na camada oculta e de saída, número de ciclos de treinamento e configurações dos algoritmos. Os pesos iniciais de todas as redes foram gerados aleatoriamente. Para cada combinação avaliada gerou-se 100 redes, sendo considerada uma simulação. A escolha da melhor configuração e o processo de treinamento das redes neurais artificiais foi feito avaliando o erro quadrático médio e o desvio padrão dos erros quadráticos médios de cada simulação, sendo escolhidas as configurações mais simples e consistentes. Como critério de parada dos algoritmos de treinamento, utilizou-se: o número total de ciclos ou épocas, que representam a quantidade de vezes que os dados são apresentados ao algoritmo (com os valores variando de 500 a 3000 (a cada 100 ), totalizando 26 valores) ou o erro quadrático médio inferior a $1 \%$. Portanto, finalizou-se o treinamento quando um dos critérios foi atingido.

O treinamento e a avaliação das redes foram feitos no sistema NeuroForest 3.0, disponível gratuitamente no site http://neuroforest.ucoz.com/, a versão disponível no site foi alterada, a fim de rodar scripts executando as configurações testadas nesse trabalho.

\section{Avaliação das Redes Neurais Artificiais}

As estimativas das RNA foram avaliadas com base nos coeficientes de correlação, com os valores observados e a raiz quadrada do erro médio percentual (RMSE), bem como na análise gráfica dos resíduos (erros percentuais) e histograma de frequência percentual dos erros percentuais (CAMPOS; LEITE, 2013).

$$
\operatorname{RMSE}(\%)=100 \sqrt{\frac{\sum_{i=1}^{n}\left(Y_{i}-\hat{Y}_{i}\right)^{2}}{n}} / \bar{Y}
$$

em que, Y é a saída observada nos inventários, $\widehat{Y}$ é a saída estimada, $\bar{Y}$ é a média da saída observada e $n$ é o número total de dados.

\section{RESULTADOS E DISCUSSÃO}

Foram avaliadas 200 configurações diferentes de RNA referentes ao número de neurônios na camada oculta e combinações de funções de ativação. Como critério de parada, utilizou-se o número de ciclos, totalizando 26 critérios. Essas configurações foram combinadas com as simulações de cada algoritmo backpropagation (8.004.000), resilient propagation (4), Manhattan update rule (1), scaled conjugate gradiente (1), levenberg marquardt (1), quick propagation (100), simulated annealing (10), e algoritmos genéticos (114). No total, foram testadas $4,1610^{10}$ configurações, sendo treinadas $4,1610^{12} \mathrm{RNA}$.

Em função da enorme quantidade de redes treinadas, foram avaliadas apenas as configurações que apresentaram valores satisfatórios de erro quadrático médio, ou seja, valores menores que 1\%.

Obteve-se resultados satisfatórios somente com configurações superiores a 4 neurônios na camada oculta, demonstrando a complexidade e não linearidade apresentada pelo modelo. O número de neurônios na camada oculta é responsável, principalmente, pela extração de características de não linearidade dos dados, neste caso, um número superior a quatro neurônios é necessário para a extração da relação entre as variáveis consideradas e a densidade básica.

Recomenda-se que, nas aplicações de RNA, sejam escolhidas configurações mais simples, com o menor número de neurônios possível na camada oculta, a fim de evitar o overfitting e facilitar o processo de busca e otimização da configuração para uma determinada tarefa (BULLINARIA, 2010).

Não houve diferença entre as funções de ativação na camada oculta avaliadas. Para as funções de ativação na camada de saída, a função linear, log seno e tangente hiperbólica apresentaram valores negativos para algumas estimativas de densidade, devido à normalização das variáveis numéricas ter sido feita entre valores de 0 a 1 , enquanto que estas funções resultam em valores entre -1 e 1 .

Não houve redes satisfatórias com os algoritmos scaled conjugate gradiente, Manhattan update rule, levenberg marquardt, simulated annealing e algoritmos genéticos, para os dados desse estudo em nenhuma configuração testada. 
Para taxas de aprendizado, com valores acima de 0,1, o algoritmo backpropagation mostrou-se instável, não convergindo. Valores baixos de taxa de aprendizagem (menores que 0,01) e do momentum (menores que 0,01) apresentaram resultados satisfatórios, não influenciando de forma perceptível, o tempo de treinamento do algoritmo, apesar de exigir um maior número de ciclos para a convergência.

Não foi objetivo avaliar o tempo de processamento, pelo fato do sistema NeuroForest 3.0 ser extremamente eficiente no processo de treinamento (cada simulação gastou aproximadamente 10 segundos), o que torna o tempo fator irrelevante nesse caso.

Para o algoritmo resilient propagation, somente as configurações com mais de três neurônios na camada oculta apresentaram resultados satisfatórios. Não houve diferença entre as quatro variações básicas RPROP+, RPROP-, iRPROP+, iPROP.

$\mathrm{O}$ algoritmo quick propagation apresentou resultados satisfatórios somente para configurações que possuíam mais de três neurônios na camada oculta e taxa de aprendizado inferior a 1,0. Taxas superiores a 1,0 causaram instabilidade no algoritmo não apresentando convergência.

Selecionou-se duas configurações que apresentaram resultados satisfatórios para demonstração gráfica (Figura 1): A) quatro neurônios na camada oculta, com função de ativação tangente hiperbólica na camada oculta e sigmoide na camada de saída, treinamento utilizando o algoritmo backpropagation com taxa de aprendizado igual a 0,001 e momentum nulo; e B) 8 neurônios na camada oculta, com função de ativação tangente hiperbólica na camada oculta e sigmoide na camada de saída, treinamento utilizando o algoritmo resilient propagation (RPROP+).

(A)

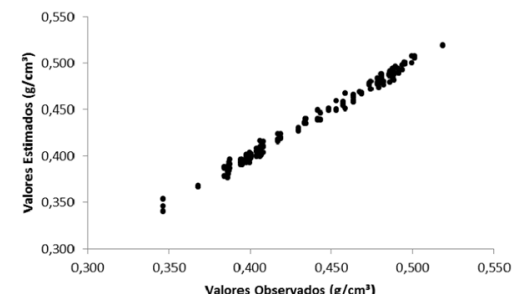

(B)

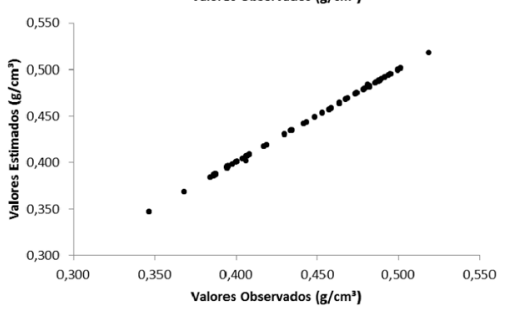

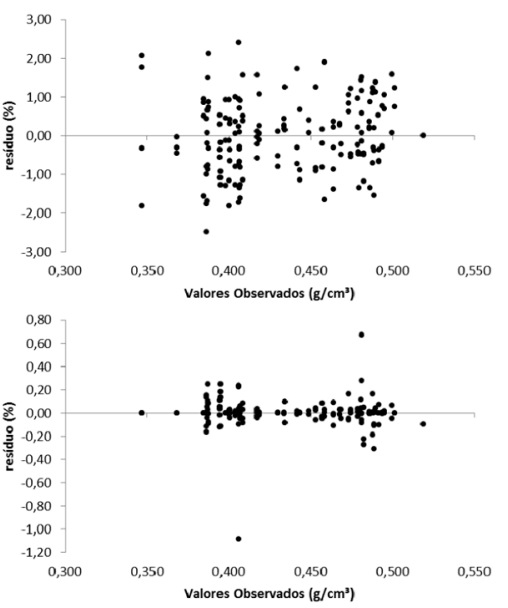

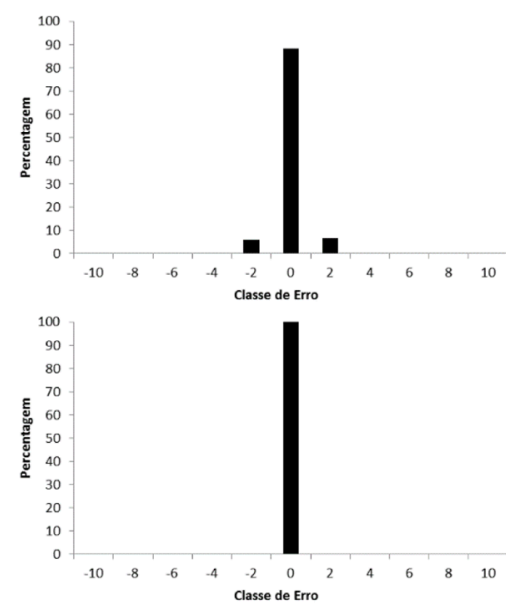

Figura 1. Relação entre valores Observados e estimados para dados de validação, Gráfico de resíduos e histograma de erros obtidos pelas estimativas realizadas pelas RNA treinadas (A e B).

Figure 1. Relationship between Observed and predicted values, and histogram graph waste error estimates obtained by the trained ANN. ( $A$ and $B$ ).

\section{CONCLUSÕES}

As redes neurais artificiais podem ser utilizadas para a estimação da densidade básica em função de valores obtidos no inventário florestal.

\section{REFERÊNCIAS BIBLIOGRÁFICAS}

BELINI, U. L. Caracterização e alterações na estrutura anatômica da madeira do Eucalyptus grandis em três condições de desfibramento e efeito nas propriedades tecnológicas de painéis MDF. 2007. 89 p. Dissertação (Mestrado em Tecnologia de Produtos Florestais) Escola Superior de Agricultura "Luiz de Queiroz". Universidade de São Paulo, Piracicaba, 2007.

BINOTI, D. H. B. Estratégias de regulação de florestas equiâneas com vistas ao manejo da paisagem. 2010, 145 p. Dissertação (Mestrado em Ciência Florestal) - Universidade Federal de Viçosa, Viçosa, 2010.

BULLINARIA, J. A. Introduction to neural computation: notas de aula. 2010. Disponível em: < http://www. cs.bham.ac.uk/ jixb/inc.html >. Acesso em: 10 dez. 2010. 
Leite et al. - Redes Neurais Artificiais para a estimação da densidade básica da madeira

CAMPOS, J. C. C.; LEITE, H. G. Mensuração florestal: perguntas e respostas. 4.ed. Viçosa: UFV, 2013.548 p.

DIAS, F. M.; LAHR, F. A. R. Estimativa de propriedades de resistência e rigidez da madeira através da densidade aparente. Scientia Forestalis, Piracicaba, n. 65, p. 102-113, 2004.

GOLDSCHIMIDT, R.; PASSOS, E. Data mining: um guia prático. Rio de Janeiro: Campus, 2005.

HEATON, J. Programming Neural Networks with Encog3 in Java. 2.ed. Heaton Research, Incorporated, 2011. 240 p.

KNAPIC, S.; LOUZADA, J. L.; LEAL, S.; PEREIRA, H. Radial variation of wood density components and ring width in cork oak trees. Annals of Forest Science, Nancy, v. 64, n. 2, p. 211-218, 2007.

LEITE, H. G.; SILVA, M. L. M.; BINOTI, D. H. B.; FARDIN, L.; TAKIZAWA, F. H. Estimation of inside-bark diameter and heartwood diameter for Tectona grandis Linn. trees using artificial neural networks. European Journal of Forest Research, Berlin, v. 130, n. 2, p. 263-269, 2010.

MITCHELL, H. L. Wood quality evaluation from increment cores. Tappi Journal, Atlanta, v. 41, n. 4, p. 150$156,1958$.

MOKFIENSKI, A.; COLODETTE, J. L.; GOMIDE, J. L.; CARVALHO, A. M. M. L. A importância relativa da densidade da madeira e do teor de carboidratos no rendimento de polpa e na qualidade do produto. Ciência Florestal, Santa Maria, v. 18, n. 3, p. 401-413, 2008. PANSHIN, A.J.; DE ZEEUW, C. Textbook of wood technology. 4.ed. New York: Mcgraw-Hill, 1980. 722 p.

SANTOS, S. R.; SANSÍGOLO, C. A. Influência da densidade básica da madeira de clones de Eucalyptus grandis x Eucalyptus urophylla na qualidade da polpa branqueada. Ciência Florestal, Santa Maria, v. 17, n. 1, p. 5363, jan./mar. 2007.

SILVA, M. L. M. Estimação do diâmetro sem casca e diâmetro do cerne para árvores de Tectona grandis Linn. utilizando redes neurais artificiais. 2008. 47 p. Monografia (Trabalho de Conclusão do Curso de Engenharia Florestal) - Universidade Federal de Viçosa, Viçosa, 2008.

TSOUMIS, G. Science and technology of wood: Structure, properties, utilization. New York: Van Nostrand Reinhold, 1991. 494 p.

VITAL, R. R.; BASTOS FILHO, J. G.; VALENTE, O. F. Efeito da constituição química e da densidade da madeira de clones de Eucalyptus grandis na produção de carvão vegetal. Revista Árvore, Viçosa, v. 10, n. 2, p. 151-60, 1986

Recebido em 15/10/2014

Aceito para publicação em 12/08/2015 